\title{
Neutronics analysis of the International Fusion Materials Irradiation Facility using the PHITS code
}

\author{
Daisuke Kaku, Tao Yea , and Yukinobu Watanabe \\ Department of Advanced Energy Engineering Science, Kyushu University, Kasuga, Fukuoka 816-8580, Japan
}

\begin{abstract}
The PHITS code is applied to neutronic calculations for the high flux test module in IFMIF. The neutron energy spectrum and heating rate calculated with the $\operatorname{Li}(\mathrm{d}, \mathrm{xn})$ neutron source term obtained by the McDelicious code reproduce the FZK result fairly well. The sensitivity of three nuclear data libraries (LA150, NRG-2003, and JENDL/HE-2004) to nuclear heating calculation and the validity of KERMA approximation are discussed. A new nuclear data evaluation of the $\mathrm{d}+\mathrm{Li}$ reaction is proposed on the basis of an advanced model calculation with a continuum-discretized coupled-channels (CDCC) method. A preliminary CDCC analysis of deuteron elastic scattering from $\mathrm{Li}$ is presented.
\end{abstract}

\section{Introduction}

The International Fusion Materials Irradiation Facility (IFMIF) [1] is composed of an accelerator-driven deuteronlithium neutron source for irradiation tests of fusion reactor candidate materials. Neutrons up to about $55 \mathrm{MeV}$ will be produced by two $125 \mathrm{~mA}$ beams of $40 \mathrm{MeV}$ deuterons bombarding a thick target of flowing liquid lithium. So far, nuclear design of IFMIF has been performed mainly using a code McDelicious developed in Forschungszentrum Karlsruhe (FZK) [2-4]. In future detailed design of IFMIF, more accurate estimation will be required on behaviors of fast neutrons with energies up to $55 \mathrm{MeV}$ in materials. Several high-energy particle transport codes, such as MCNPX [5] and PHITS [6], are widely used for various accelerator applications in combination with current high energy nuclear data libraries. Therefore, it is worthwhile to test to what extent these codes and high energy nuclear data libraries are applicable to IFMIF neutronic calculations.

We have chosen the PHITS code for calculations of nuclear heating in the high flux test module (HFTM) in IFMIF, which is required for thermal-hydraulic design of HFTM [7]. The purpose of the present work is to examine the applicability of the PHITS code to IFMIF neutronic calculations. Neutron energy spectrum and nuclear heating over the HFTM are calculated and compared with FZK results. Furthermore, the sensitivity of three nuclear data libraries (LA150 [8], NRG2003 [9], and JENDL/HE-2004 [10]) to nuclear heating and the validity of KERMA approximation are discussed.

In the PHITS code, the quantum molecular dynamics (QMD) [11] plus generalized evaporation model (GEM) [12] is employed to deal with nuclear reactions that may occur in heavy-ion transport processes in matter. We applied the PHITS code to a preliminary calculation of the $\operatorname{Li}(\mathrm{d}, \mathrm{xn})$ neutron source term. However, the result could not reproduce satisfactorily both experimental data of thick target yields [13] and a prediction obtained by the McDelicious code [3]. Thus, it turned out that the present version of QMD is not appropriate for deuteron-induced reactions. On the other hand, there are

\footnotetext{
${ }^{a}$ Presenting author, e-mail: yetao@aees. kyushu-u.ac.jp
}

still some discrepancies between McDelicious calculations and the latest measurement [13] for double-differential thicktarget yields and double-differential $\mathrm{Li}(\mathrm{d}, \mathrm{xn})$ cross sections. In addition, the latest version ENDF/B-VII [14] is not enough to use IFMIF nuclear design, because it contains only the deuteron data for ${ }^{6,7} \mathrm{Li}$ for incident energies up to $5 \mathrm{MeV}$ and $20 \mathrm{MeV}$, respectively. These situations have initiated a new nuclear data evaluation of the $\mathrm{Li}(\mathrm{d}, \mathrm{xn})$ reaction using theoretical model calculations based on a continuum-discretized coupled channels (CDCC) method [15,16] suitable for description of deuteron breakup and stripping processes. A preliminary CDCC result is presented and compared with experimental data in this paper.

\section{Application of PHITS code to IFMIF neutronics}

\subsection{Calculation procedure}

The high flux test module (HFTM) is placed downstream behind the flowing liquid lithium target, forming the highest neutron radiation region. The HFTM consists of a steel container housing a number of irradiation rigs that contain encapsulated irradiation specimens.

The PHITS code is used for IFMIF-HFTM neutronic calculations. The details of the PHITS code are described elsewhere [6].

In the present calculation, a simplified HFTM configuration is adopted as in the previous FZK work [2]. The geometrical configuration is depicted in figure 1. The HFTM part is composed of a rectangular block $20 \times 5 \times 5 \mathrm{~cm}^{3}$ filled with Eurofer with a mass density of $6.24 \mathrm{~g} / \mathrm{cm}^{3}$, which is $80 \%$ of the normal density to take account of the space occupied by cooling gas.

Each of two deuteron beams impinges on the lithium target with $10^{\circ}$ declination angle in vertical direction. Figure 1 illustrates the lithium target $\left(26 \times 2.5 \times 20 \mathrm{~cm}^{3}\right)$ filled with lithium at $0.512 \mathrm{~g} / \mathrm{cm}^{3}$ and its back plate $\left(26 \times 0.18 \times 20 \mathrm{~cm}^{3}\right)$ filled with Eurofer at $7.8 \mathrm{~g} / \mathrm{cm}^{3}$ density. The PHITS code can calculate neutron production from the $\operatorname{Li}(\mathrm{d}, \mathrm{xn})$ reaction in the lithium target using the QMD model [11] in principle, but a 


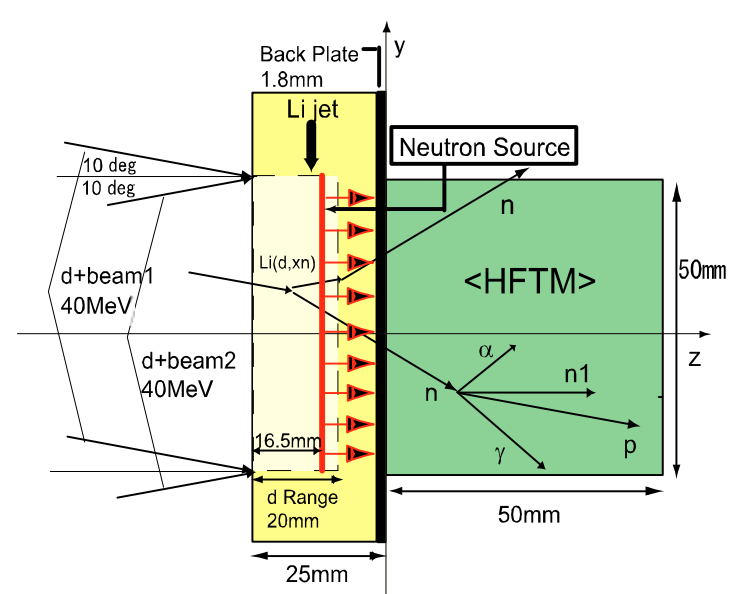

Fig. 1. Geometrical configuration of the lithium target and HFTM.

preliminary result does not show reasonable agreement with experimental results. Thus, the differential thick target neutron yields (TTY) calculated by the McDelicious code [3] are used as the source term in the present work. The neutron source term is assumed to be a surface source placed at a distance $16.5 \mathrm{~mm}$ from the entrance surface, so that the calculated average neutron flux in the HFTM reproduces well the FZK result given in table 1 . The tilt angles of $\pm 10^{\circ}$ of incident deuteron beam are taken into account so that the direction of neutron emission at $0^{\circ}$ coincides with that of the incident deuteron beam. It should be noted that scattering of neutrons from the lithium layer between the surface neutron source and the back plate is neglected because the calculated TTY data are used as the neutron source term.

\subsection{Results and discussion}

Energy distribution of the average neutron flux in the HFTM calculated by the PHITS code with LA150 is compared with the FZK result obtained by the McDelicious code in figure 2 . The former reproduces the latter well, although there is a slight difference in the energy range between 10 and $25 \mathrm{MeV}$. The difference might be due to that in the neutron source term, because the surface neutron source is assumed in the present work as mentioned above. Table 1 shows comparisons of the average neutron and gamma fluxes, the average dpa rate, the total heat production, and the average heat production density. The present calculation shows fairly good agreement with the FZK result. Thus, this benchmark test indicates that the PHITS code will be applicable to neutronic calculations related to thermal-hydraulic design of the HFTM.

The spatial distribution of nuclear heating rate in the HFTM is calculated for the case where each of two deuteron beams $(2 \times 125 \mathrm{~mA})$ impinges on the lithium target with $10^{\circ}$ declination angle in horizontal direction. It should be noted that the beam incidence with horizontal declination is adopted in the latest IFMIF design [4]. In figure 3, the result is presented as a three-dimensional plot sliced in half at $\mathrm{x}=0$. The size of each voxel (i.e., an elementary cubic segment) is

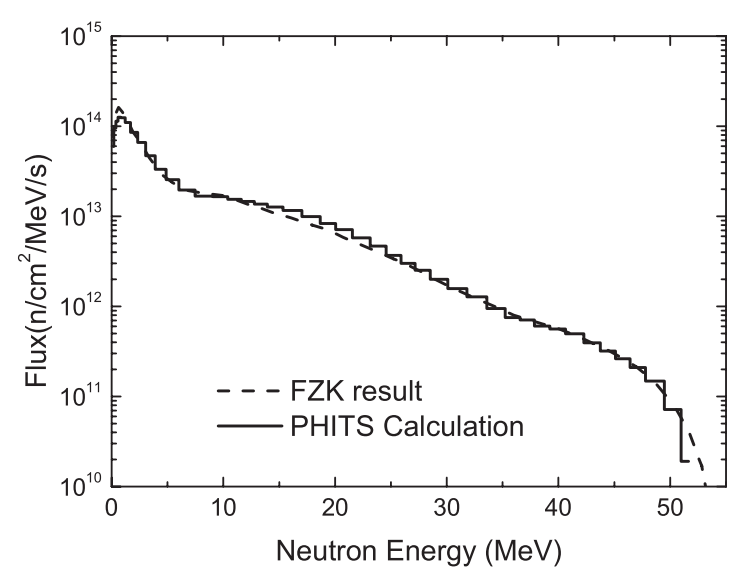

Fig. 2. Comparison of neutron energy spectra in the HFTM between the present PHITS calculation and the FZK result [2].

Table 1. Physical parameters of the HFTM and results of neutronics calculations. The result of the FZK work is take from ref. [2] for comparison.

\begin{tabular}{lcc}
\hline \multicolumn{1}{c}{ Parameter } & FZK work & Present work \\
\hline Ave. neutron flux $\left(\mathrm{n} / \mathrm{cm}^{2} / \mathrm{s}\right)$ & $5.86 \times 10^{14}$ & $5.89 \times 10^{14}$ \\
Ave. neutron energy $(\mathrm{MeV})$ & 7.0 & 7.0 \\
Ave. $\gamma$-ray flux $\left(\gamma / \mathrm{cm}^{2} / \mathrm{s}\right)$ & $2.33 \times 10^{14}$ & $2.43 \times 10^{14}$ \\
Ave. dpa rate $(\mathrm{dpa} / \mathrm{fpy})$ & 29 & 29 \\
Total heat production $(\mathrm{kW})$ & 7.0 & 7.0 \\
Ave. heat prod. density $\left(\mathrm{W} / \mathrm{cm}^{3}\right)$ & 14.0 & 14.0 \\
\hline
\end{tabular}

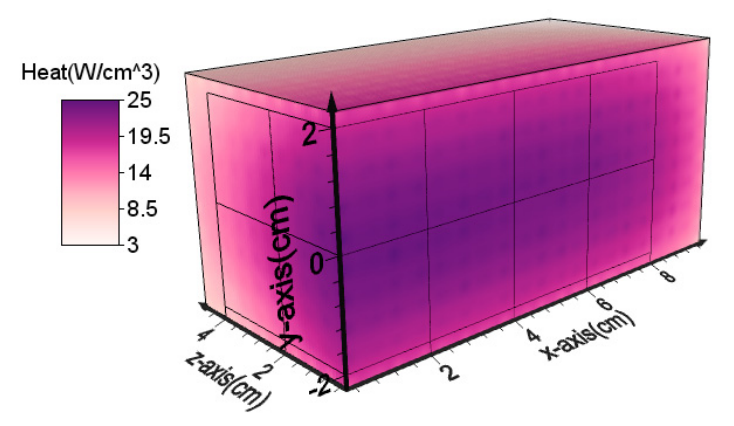

Fig. 3. Three-dimensional spatial distribution of nuclear heating rate calculated by assuming deuteron beam incidence with horizontal declination.

$0.5 \times 0.5 \times 0.5 \mathrm{~cm}^{3}$. The highest heating rate of $25 \mathrm{~W} / \mathrm{cm}^{3}$ is obtained in the vicinity of the surface region.

The neutronic calculations are performed using the PHITS code with three nuclear data libraries (LA150 [8], NRG2003 [9], and JENDL/HE-2004 [10]) in order to see how nuclear data libraries affect calculations of neutron flux and nuclear heating. The calculated neutron fluxes are almost identical among three calculations, while the total heating rates are largely different (up to 50\%) as shown in table 2. It is found that the difference in the total heating rates is due to that in the heat generated by neutrons. This can be easily explained from the fact that the heating numbers of ${ }^{56} \mathrm{Fe}$ (i.e., kerma factors) included in the libraries are 


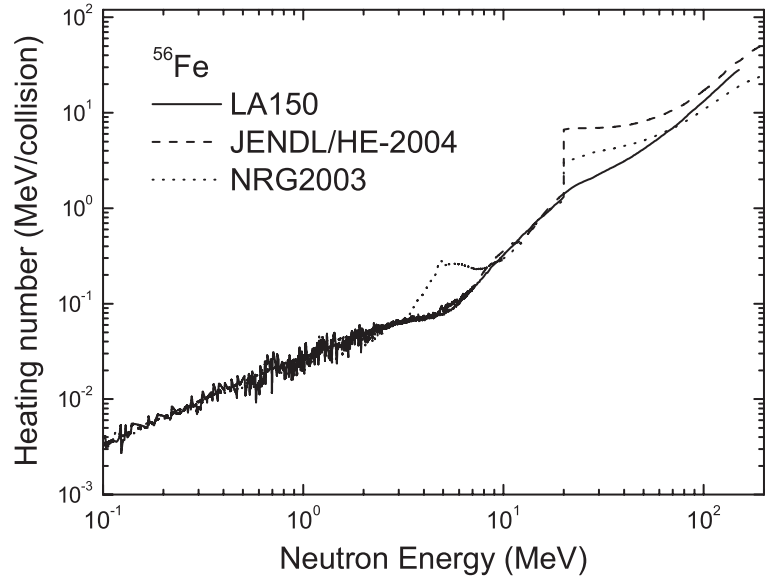

Fig. 4. Total heating number of ${ }^{56} \mathrm{Fe}$.

Table 2. Comparison of calculated heating rates among three nuclear data libraries (LA150, JENDL/HE-2004, and NRG-2003).

\begin{tabular}{lccc}
\hline \multicolumn{1}{c}{ Heating rate } & LA150 & JHE-2004 & NRG-2003 \\
\hline Total heating rate $(\mathrm{kW})$ & 7.0 & 10.6 & 8.0 \\
Neutron heating rate $(\mathrm{kW})$ & 3.1 & 6.4 & 4.2 \\
Photo heating rate $(\mathrm{kW})$ & 3.9 & 4.2 & 3.8 \\
Ratio to LA150 & 1.0 & 1.5 & 1.1 \\
\hline
\end{tabular}

obviously different, particularly in the high energy range above $20 \mathrm{MeV}$, as seen in figure 4 .

As neutron energy increases, production of light ions, such as protons and deuterons with relatively high kinetic energy, becomes prominent. With an increase in the kinetic energy, the range becomes long, e.g., the range of $50 \mathrm{MeV}$ protons is $5.3 \mathrm{~mm}$ in Eurofer with the mass density $6.24 \mathrm{~g} / \mathrm{cm}^{3}$. Therefore, it is expected that the KERMA approximation assuming local energy deposition becomes worse. The PHITS code has a feature to deal with transport of light ions in matter using the continuous slowing down approximation. This means that the spatial spreading of the energy deposited by light ions can be taken into account beyond the KERMA approximation in the present calculation. It should be noted that the KERMA approximation is adopted for heavy recoils because the range is very short. In the preceding work in FZK [2], the KERMA approximation was used in calculations of heat production. Therefore, it is of interest to examine quantitatively the validity of the KERMA approximation in the HFTM design.

The calculation condition is same as mentioned in subsect. 2.1, except that neutrons enter in the direction perpendicular to the surface xy-plane of the HFTM. The PHITS calculation is implemented under the full KERMA approximation with the total heating numbers included in the nuclear data library, which is called the PHITS-KERMA calculation in figure 5, and compared with the normal PHITS calculation mentioned above. The spatial distributions of total heat production are compared between the two cases in figure 5. One can see that the difference appears slightly in the vicinity of the surface (up to about $1 \mathrm{~mm}$ ), because most of generated light ions are expected to escape from the front surface.

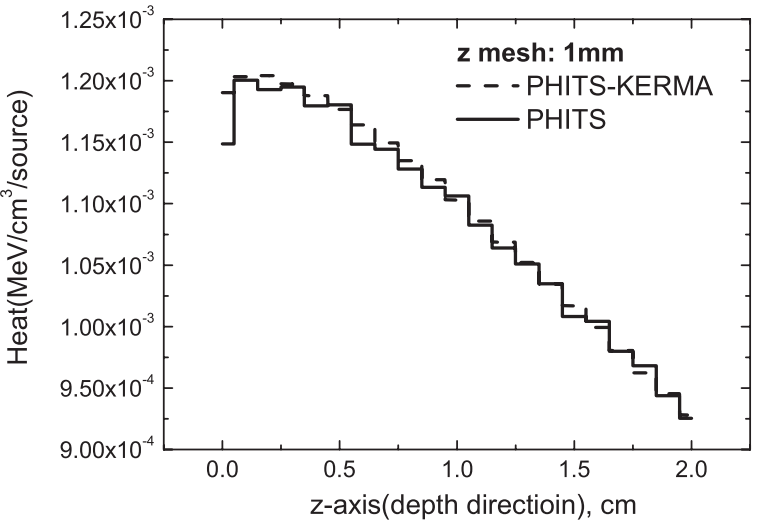

Fig. 5. Spatial distribution of total heat production.

\section{Evaluation of $d+$ Li cross-section data using CDCC method}

\subsection{CDCC method}

Since a deuteron is a loosely-bound nucleus composed of a neutron and a proton, a deuteron breakup is expected to contribute to the $\operatorname{Li}(\mathrm{d}, \mathrm{xn})$ reaction if the incident energy is larger than its binding energy. The CDCC method $[15,16]$ can deal with the deuteron breakup process using a phenomenological three-body hamiltonian in which the nucleon-nucleus interaction is represented by the optical model potential (OMP) at half the deuteron incident energy and an effective nucleon-nucleon potential is used for the p-n interaction. Recently, the CDCC calculation has been successfully applied for the $(\mathrm{d}, \mathrm{n})$ reaction on ${ }^{7} \mathrm{Be}$, which is a mirror nucleus of ${ }^{7} \mathrm{Li}$, at $8 \mathrm{MeV}$ [17]. Thus, the CDCC method is expected to be a promising approach to describe the $\mathrm{Li}(\mathrm{d}, \mathrm{xn})$ reaction.

In the present work, the CDCC code [18] is used to analyze deuteron elastic scattering from ${ }^{6,7} \mathrm{Li}$ as the first stage to confirm the applicability. In the analyses, the spin of deuteron, $S=1$, is considered and both the central and spin-orbit terms of the nucleon optical potentials are included. The deuteron ground state wave function is composed of ${ }^{3} S_{1}$ state alone, and the mixing of $D$-states in it is neglected. The breakup state wave functions contains the relative angular momentum of the p-n subsystem $l=0$ and 2, i.e., ${ }^{3} S_{1},{ }^{3} D_{1},{ }^{3} D_{2}$, and ${ }^{3} D_{3}$. The ground-state and breakup-state wave functions are constructed using Gaussian potentials [16]. The maximum linear momentum of the p-n subsystem and the total number of the discretized continuum bins are set to be $1.2 \mathrm{fm}^{-1}$ and 20 , respectively.

\subsection{Result of deuteron elastic scattering}

A preliminary result is presented in figure 6 for deuteron elastic scattering from ${ }^{7} \mathrm{Li}$ at $28 \mathrm{MeV}$ together with experimental data [19]. Neutron and proton OMPs are taken from refs. [22, 23], respectively, because the optical model calculations with the OMPs predict successfully differential cross sections of nucleon elastic scattering from ${ }^{7} \mathrm{Li}$ at $14 \mathrm{MeV}$ corresponding to half of the deuteron incident energy. As shown in figure 6, 


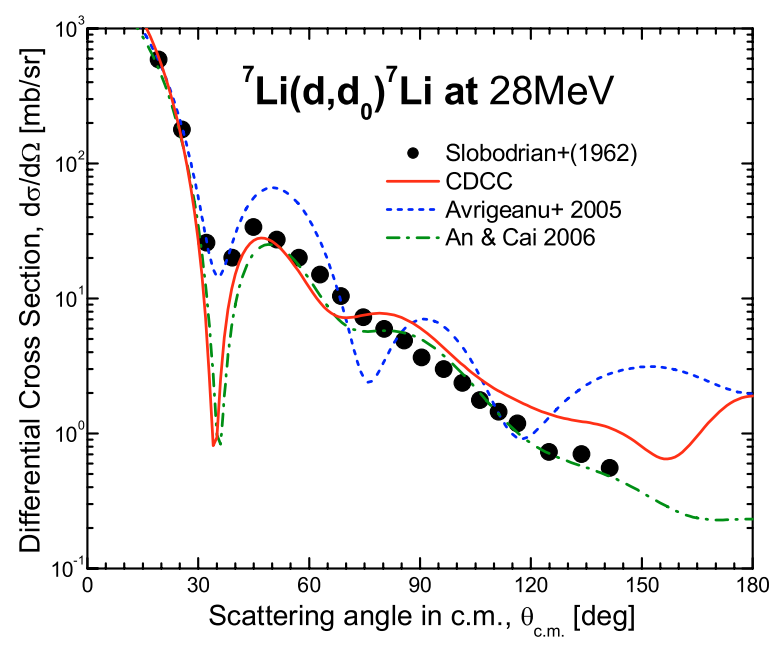

Fig. 6. Differential cross sections of deuteron elastic scattering from ${ }^{7} \mathrm{Li}$ at $28 \mathrm{MeV}$.

the CDCC calculation reproduces the measurement satisfactorily well to the same extent as deuteron optical model calculations using deuteron OMPs [20,21], except around the first minimum and at backward angles. Similar CDCC analyses were extended to other energies and a target ${ }^{6} \mathrm{Li}$. For low incident energies, it was found that the CDCC calculations do not necessarily reproduce experimental data well, especially at backward angles, as shown in ref. [17]. This might be improved by taking account of compound elastic scattering.

\section{Summary and conclusions}

The PHITS code was first applied to neutronic calculations for the high flux test module (HFTM) in the IFMIF neutron source facility. The calculated neutron energy spectrum and nuclear heating were in good agreement with the previous result by the FZK group. This indicates the applicability of the PHITS code to neutronic calculations for the HFTM. The calculation using different nuclear data libraries, LA150, NRG-2003, and JENDL/HE-2004, showed that the neutron fluxes are almost identical, while the heating rates have a large discrepancy, reflecting the difference in the heating numbers included in these libraries. In addition, it was confirmed that the KERMA approximation is reasonably good in calculating the heating rates in the HFTM unless one discusses the heat generated within the small size less than $1 \mathrm{~mm}$.

It is necessary to improve the $\operatorname{Li}(\mathrm{d}, \mathrm{xn})$ neutron source term modelling in order to upgrade IFMIF neutronic calculations using the PHITS code. We have proposed a new nuclear data evaluation of the $\mathrm{Li}(\mathrm{d}, \mathrm{xn})$ reaction using the continuumdiscretized coupled channels (CDCC) method. As the initial phase, the CDCC calculation was applied to deuteron elastic scattering from ${ }^{6,7} \mathrm{Li}$. A choice of appropriate nucleon optical potentials was found to lead to overall good agreement with the experimental data. This success encourages us to apply the CDCC method to prediction of neutron production from the deuteron breakup process using breakup $S$-matrix elements by following Iseri's prescription for (d,pn) reactions [16].

The authors wish to thank K. Niita for his kind instruction on use of the PHITS code. They acknowledge K. Kosako for his useful discussion on the heating numbers in nuclear data libraries. They are also grateful to $\mathrm{K}$. Ogata for his valuable advice on CDCC calculations. This work is partly supported by the Grants-in-Aid (No. 19560844) for Scientific Research from the Japan Society for the Promotion of Science (JSPS).

\section{References}

1. H. Matsui, in Proceedings of the 23rd Symposium on Fusion Technology, Venice, Italy, Sept. 20-24, 2004.

2. S.P. Simakov et al., Forschungszentrum Karlsruhe Report FZKA 6743 (2002).

3. S.P. Simakov et al., J. Nucl. Mater. 307-311, 1710 (2002).

4. U. Fischer et al., Fus. Eng. Des. 81, 1195 (2006).

5. L.S. Waters (ed.), MCNPX Uers's Manual, Version 2.3.0, Los Alamos National Laboratory Report LA-UR-02-2607 (2002).

6. H. Iwasa, K. Niita, T. Nakamura, J. Nucl. Sci. Technol. 39, 1142 (2002).

7. S. Ebara et al., Fus. Eng. Des. 81, 887 (2006); S. Ebara et al., Fus. Eng. Des. 82, 61 (2007).

8. M.B. Chadwick et al., Nucl. Sci. Eng. 131, 293 (1999); LA-UR99-1222 (1999).

9. A.J. Koning et al., in Proceedings of the International Conference on Nuclear Data for Science and Technology, Santa Fe, USA, Sept. 26-Oct. 1, edited by R.C. Haight, M.B. Chadwick, T. Kawano, P. Talou (AIP, New York, 2005), p. 422.

10. Y. Watanabe et al., ibid., p. 326.

11. K. Niita et al., Phys. Rev. C 52, 2620 (1995).

12. S. Furihata, Nucl. Instrum. Meth. Phys. Res. B 171, 251 (2000).

13. M. Hagiwara et al., J. Fus. Sci. Technol. 48, 1320 (2005).

14. M.B. Chadwick et al. (CSEWG collaboration), Nucl. Data Sheets 107, 2931 (2006).

15. M. Yahiro et al., Prog. Theor. Phys. Suppl. 89, 32 (1986).

16. Y. Iseri et al., Prog. Theor. Phys. Suppl. 89, 84 (1986).

17. K. Ogata et al., Phys. Rev. C 67, 011602(R) (2003).

18. Y. Iseri et al., CDCDEU code, Bulletin of Research Computer System, Computing and Communications Center of Kyushu University, Vol. 5, No. 3 (2006), p. 117; HICADEU code (to be published).

19. R.J. Slobodrian, Phys. Rev. 126, 1059 (1962).

20. M. Avrigeanu et al., Nucl. Phys. A 759, 327 (2005).

21. H. An, C. Cai, Phys. Rev. C 73, 054605 (2006).

22. J.H. Dave, C.R. Gould, Phys. Rev. C 28, 2212 (1983).

23. N. Koori et al., JEARI-M 89-167 (1989). 